\title{
Diagnosis of arrhythmia based on ECG analysis using CNN
}

\author{
Muayed S. Al-Huseiny, Noor Khudhair Abbas, Ahmed S Sajit
}

Department of Electrical Engineering, College of Engineering, Wasit University, Iraq

\begin{tabular}{|c|c|}
\hline Article Info & ABSTRACT \\
\hline & \multirow{10}{*}{$\begin{array}{l}\text { Arrhythmia is the prime indicator of serious heart issues, and, hence, } \\
\text { it is essential to be detected properly for early phase treatment. This article } \\
\text { presents an approach for the diagnosis of cardiac disorders via } \\
\text { the recognition of } 17 \text { types of arrhythmia. The proposed approach includes } \\
\text { building a convolution neural network (2D-CNN) which is trained by using } \\
\text { images of Electrocardiograph (ECG) signals collected from the MIH-BIH } \\
\text { database. The ECGs are first converted into images. This step serves } \\
\text { twofold: first, CNN is best suited for classifying image data and thus reduces } \\
\text { preprocessing, and second, most ECG recordings are still being produced } \\
\text { on thermal paper which can then be captured as image. Next, 2D-CNN } \\
\text { is trained and validated. Test results show that the proposed method achieves } \\
\text { classification accuracy of } 96.67 \% \text { and error of } 0.004 \% \text {. in addition to } \\
\text { the superior accuracy achieved by this method compared to the previous } \\
\text { literature, this approach enjoys reduced processing time and complexity } \\
\text { apart from the training phase, also by dealing with images this method } \\
\text { offers high degree of versatility and can be integrated as utility within } \\
\text { other applications or wearables. }\end{array}$} \\
\hline Received Oct 15, 2019 & \\
\hline Revised Dec 30, 2019 & \\
\hline Accepted Feb 3, 2020 & \\
\hline Keywords: & \\
\hline Biomedical engineering & \\
\hline Classification of arrhythmia & \\
\hline Convolutional neural network & \\
\hline Deep learning & \\
\hline Electrocardiogram & \\
\hline
\end{tabular}

This is an open access article under the CC BY-SA license.

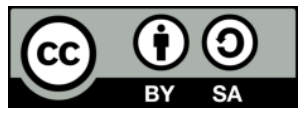

\section{Corresponding Author:}

Muayed S. AL-Huseiny,

Department of Electrical Engineering,

College of Engineering, Wasit University,

Al-Rabie Dist., Al-Kut, Wasit, Iraq.

Email: malhuseiny@uowasit.edu.iq

\section{INTRODUCTION}

Cardiovascular problems are the main challenge to healthcare providers worldwide. They are known for prevalence and related high mortality rates. It is estimated that around 17.3 person die every year as a result of heart related problems, accounting to around $37 \%$ of all deaths worldwide [1]. This also puts enormous pressure on health services in terms of hospital costs due to the recurrent nature of this illness that requires long-term care and treatment. ECG is the abbreviation of the electrocardiograph [2, 3]. It is a technique widely used by cardiologist in the diagnosis of cardiovascular diseases. It is relatively non-invasive and otherwise an easy to utilize technique. ECG provides good insight on cardiovascular health and pathology. The main clinical symptoms of heart issues are cardiac arrhythmias which is an abnormal heart rhythm. Due to the high individual variability among patients and among different stages of disease, diagnosis of cardiac issues becomes prone to human error and may contribute in some cases to life loss.

In order to achieve accuracy and consistency of diagnosis, many experiments have been conducted in the area of machine learning to predict arrhythmias automatically [4-6]. Therefore, the problem this paper is trying to tackle is the automatic diagnosis of cardiac arrhythmia; an automatic (perhaps wearable) diagnosis system can provide early warnings to rare and infrequent issues. The aim of this research is to develop a machine learning based computation mechanism that helps to diagnose cardiac arrhythmia speedily, accurately, and reliably such that proper treatment can be issued by trained doctors. 
Furthermore, this paper proposes a technique that utilizes images of the ECG recording directly by employing image processing to digitize those recordings. This has the advantage of making the proposed technique usable with older ECG devices (particularly used in developing countries) which produce their recordings on thermal paper. Figure 1 shows the structure of a general cardiac arrhythmia classification system. First, there is the data acquisition stage where images of ECG recordings are captured (this can be skipped for digital ECG devices). Next, algorithm stages are fed with data to derive and train the network until expert level knowledge is achieved. Testing is then performed to verify accuracy and measure performance.

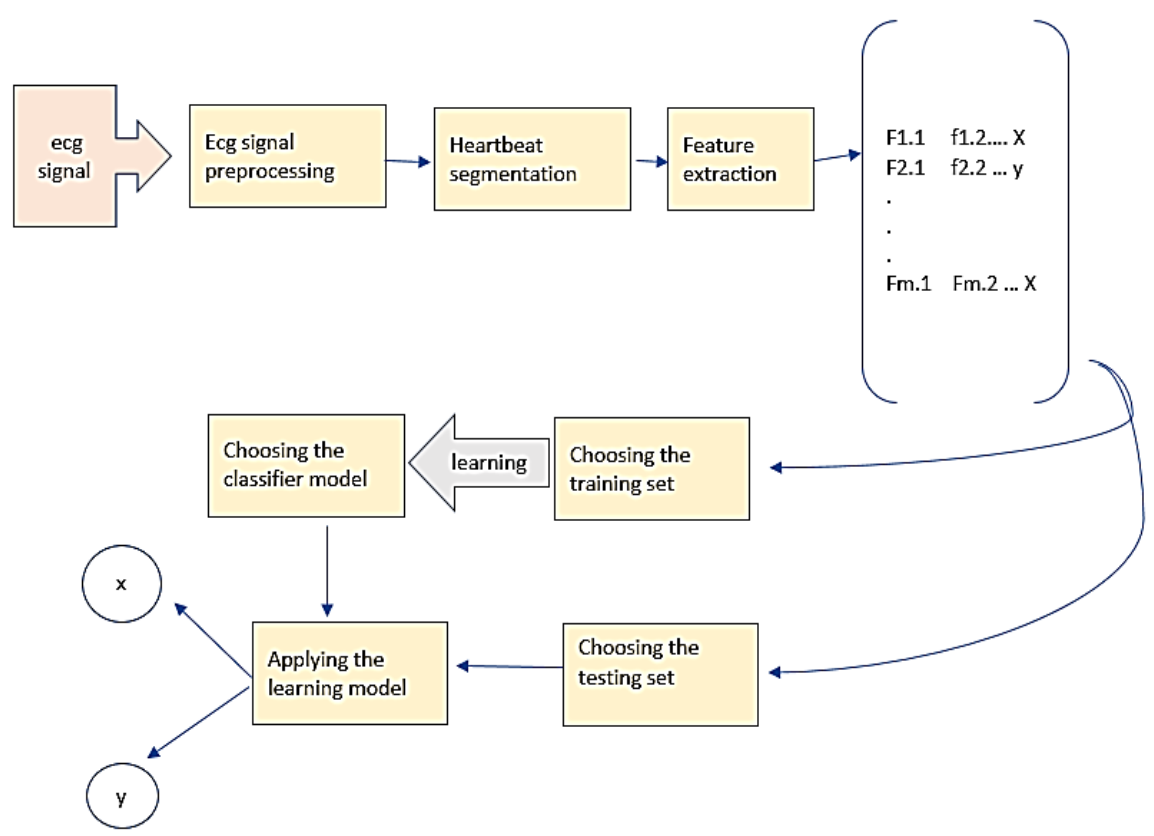

Figure 1. Detailed arrhythmia classification computing system

\section{RESEARCH METHOD}

In the proposed approach CNNs are employed to detect and classify lengthy segments of ECG recordings (10-s).in order to achieve accuracy and reduce the effects of transient fluctuations generated by acquisition equipment and other sources of noise [7]. Generally, the system of classification of heart disease using ECG signals is divided into five stages [8] (also shown in Figure 1):

- Pre-processing with normalization: The output from this stage puts data from different ECG devices and different patients in a rather standard form.

- Feature extraction: This stage isolates and strengthens signals in order to reinforce the detection of the operational dis ruptions (morphological changes) of the Electrocardiogram signal.

- Feature selection: The objective at this stage is to perform data reduction of features and thereby speed up the calculation

- Machine learning algorithms: The objective of the whole analysis is to classify sample-based cardiac illnesses (captured by ECG fragments), derived on the layout and choice of suitable variables as well as training models, and evaluated utilizing machine learning algorithms.

- Cross validation: the goal here is to reduce the impact of over-fitting of the algorithms in order to increase the generalization of the algorithm as well as increase the accuracy of the results achieved with new unseen data [9].

The proposed system utilizes 15-layer deep network architecture by using regular CNN layers. The input for this system framework (training/verification/testing data) consisted of images derived from the 360 sample/second raw Electrocardiogram signals of the MIT-BIH dataset. In comparison with the manual methods or even some computation methods, the Electrocardiogram signals were not det ected as segmented QRS complexes but rather treated as whole signals [10-12]. This reduces the complexity of the algorithm. The training and testing of system performance are carried out on the Arrhythmia database which consists of 1000 fragments representing 17 different classes of arrhythmia. It is essential to stress here 
that the proposed method, unlike previous approaches, uses 2-D image data rather than 1-D ECG signals. The experimental results show that this decision makes valuable contribution to the accuracy of the achieved results.

\subsection{Materials}

Electrocardiogram data have been obtained from PhysioNet's MIT-BIH Arrhythmia database $[13,14]$. As mentioned earlier, this Electrocardiogram data set consisted of 1000 non-overlapping frames. Each of these frames represents 10 seconds of ECG recording collected from subjects with various cardiac is sues as well as healthy one totaling 45 subjects. Those subjects are distributed as 19 women 23-89 years old and 26 men 32-89 years old. The sample fragments were registered at a $360 \mathrm{~Hz}$ sample frequency. In Table 1, a summary of the distribution of the seventeen cases included in the database is given.

Table 1. Number of ECG segments in various ECG classes

\begin{tabular}{ccc}
\hline Number & Class & Fragment Numbers (Total 1000) \\
\hline 1 & Normal sinus rhythm(NSR) & 238 \\
2 & Atrial premature beat(APB) & 66 \\
3 & Atrial flutter(AFL) & 20 \\
4 & Atrial fibrillation(AFIB) & 135 \\
5 & Supraventriculart achyarrhythmia (SVT A) & 13 \\
6 & Pre-excitation(WPW) & 21 \\
7 & Premature ventricular Contraction(PVC) & 133 \\
8 & Ventricular bigeminy & 55 \\
9 & Ventricular trigeminy & 13 \\
10 & Ventricular tachycardia(VT) & 10 \\
11 & Idioventricular rhythm(IVR) & 10 \\
12 & Ventricular flut ter(VFL) & 10 \\
13 & Fusion of ventricular and normal beat & 11 \\
14 & Left bundle branch block beat(LBBB) & 103 \\
15 & Right bundle branch block beat(RBBB) & 62 \\
16 & Second-degree heart Block(SDHB) & 10 \\
17 & Pacemaker rhythm(PR) & 45 \\
\hline
\end{tabular}

All these cases are known cardiovascular diseases, except for the normal sinus rhythm which is the case of normal heart rhythm, i.e. healthy heart condition. The table includes the name and common abbreviation of the medical condition as classified by expert cardiologists (ground truth) as well as the number of samples per condition. Figure 2 shows plotted examples of the data included in the database. In Figure 2, it is easy even for non-experts to notice the differences among difference images, however, the exact diagnosis and the case and its severity requires skill and experience which the prop osed systemaims to gather.

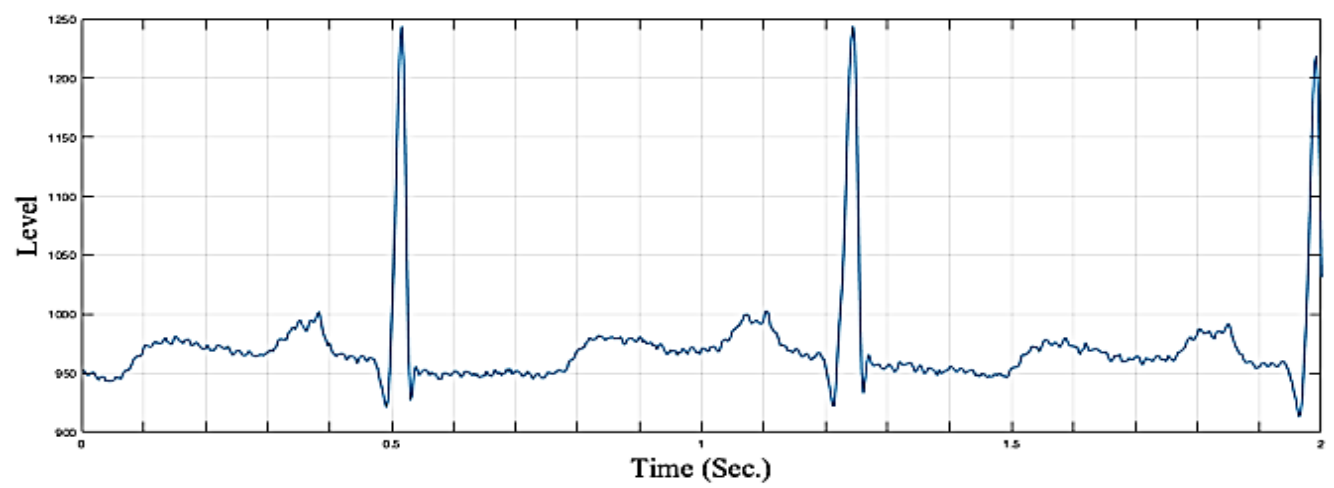

(a)

Figure 2. Actual signal samples of four different classes, (a) Normal sinus rhythm NSR 


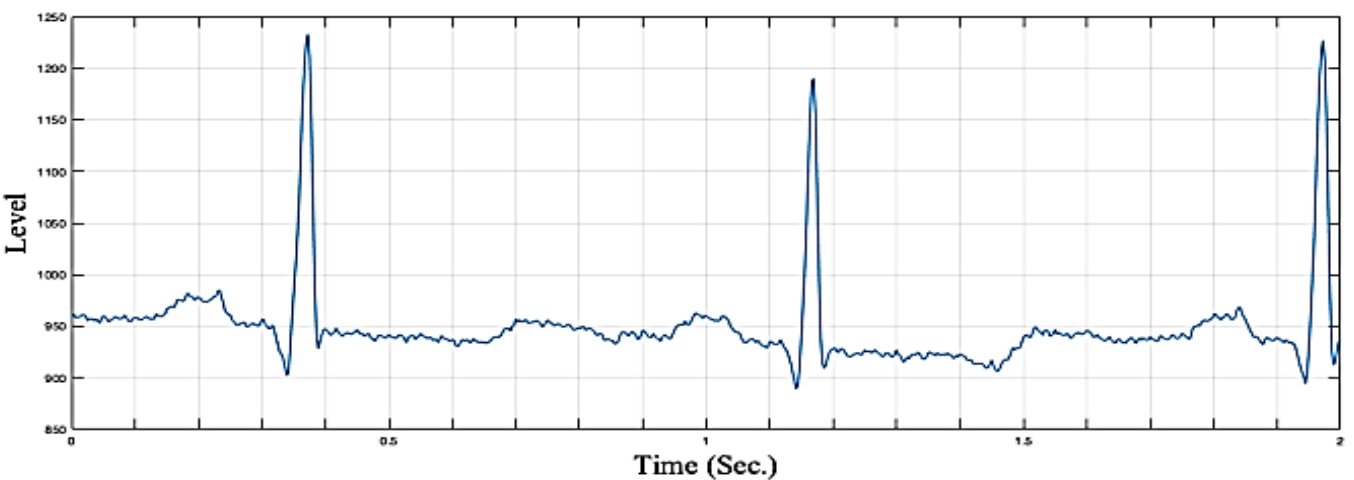

(b)

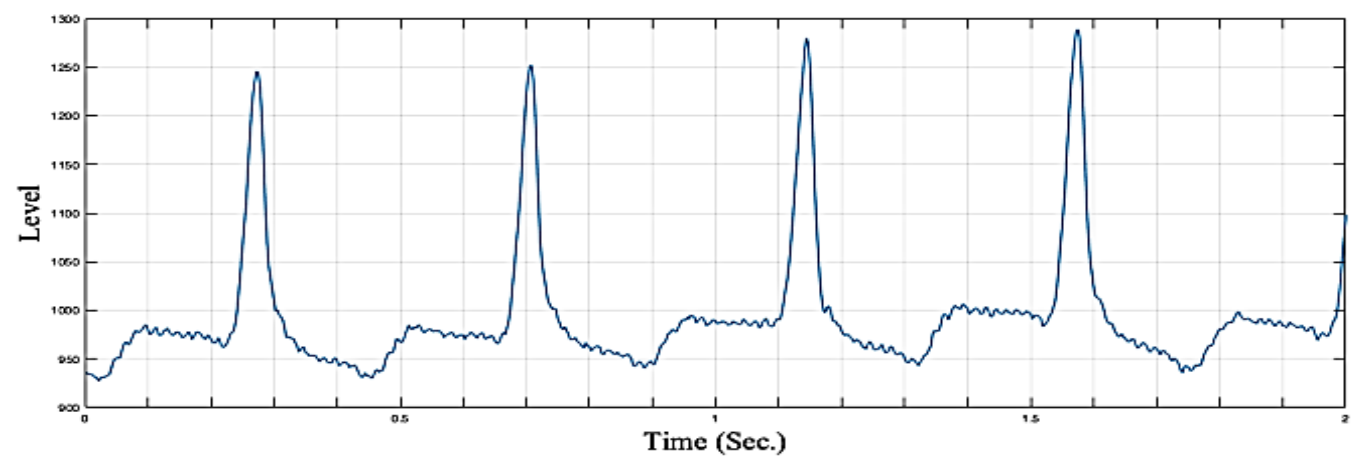

(c)

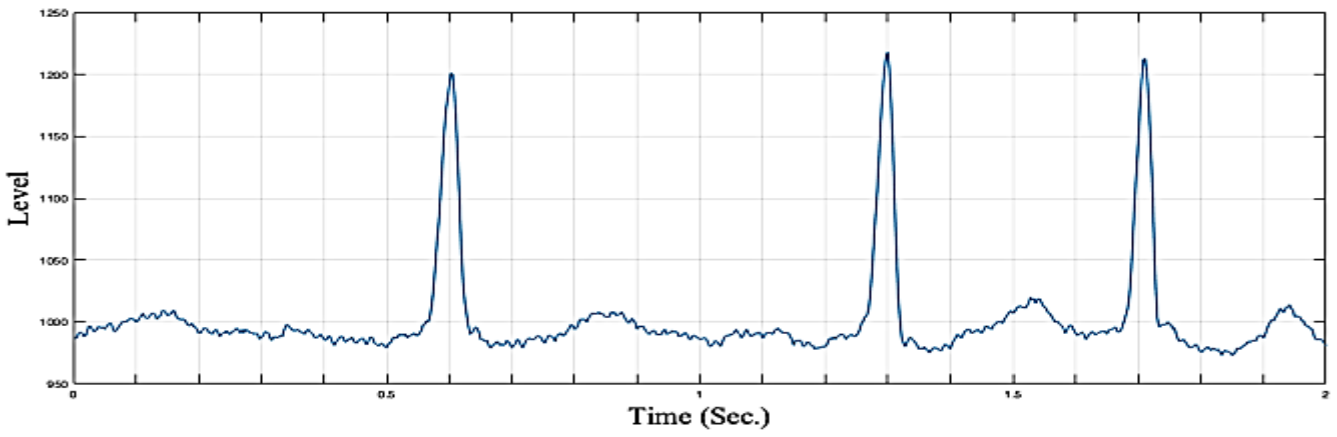

(d)

Figure 2. Actual signal samples of four different classes,(b) Atrial premature beat APB, (c) Atrial flutter AFL, (d) Atrial fibrillation AFIB (continue)

\subsection{Convolution neural network $(\mathrm{CNN})$}

Convolutional NNs or CNN is one of the types of deep learning algorithms. It was developed by K. Fukushima $[14,15]$ in the early 1980s from the original multi-layer network Perceptron. CNNs are widely utilized for machine learning, visual processing and computer vision [16, 17]. Through its several hidden layers the algorithm extracts features of a picture or video and through its fully connected layer it generates the appropriate learned output. It deals with two-dimensional and one-dimensional signals. In this article, we built a 2D CNN model. The main elements of this system are the Convolutional Layer, the Activation layer, the Pooling Layer, and the Fully Connected Layer. Activation function: is a nonlinear function that based on its input causes the firing of the node. Figure 3 shows various types of this function. Rectified Linear Unit (ReLU) is used in this work due to its many significant advantage, particularly: not triggering all neurons at the exact moment, that tends to minimize the number of simulations performed, removing negative values from activation map, and etc. 

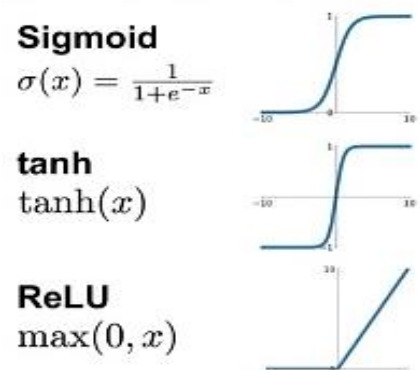

\section{Leaky ReLU \\ $\max (0.1 x, x)$}

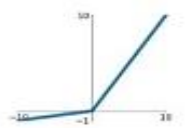

\section{Maxout}

$\max \left(w_{1}^{T} x+b_{1}, w_{2}^{T} x+b_{2}\right)$

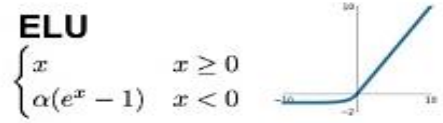

Figure 3. Various types of activation functions used in $\mathrm{CNN}$

\subsection{Operation of neural network model}

- Load new image of ECG.

- Apply Conv. Layer with 30 filters (kernel size $3 * 3$ ).

- Apply activation function (ReLU).

- Submit the output of the function to the next Conv. Layer with 50 filters.

- Apply activation function (ReLU).

- Repeat steps (2-5) 60 times (epochs) and produce trainable works.

- Feed the output to the flatten layer.

- Apply fully connected layer.

The above algorithm is applied to each image during the training phase. The stages of this algorithm are also shown in details in Figure 4. In the first stage of the algorithm, 2D convolution layer is implemented on the input ECG. After that there is the pooling layer, which basically performs down sampling operation. This help to reduce processing time, as well as to identify and define the new parameters. Next layer we have dropout layer, this layer drops out random set of responses (activations) by setting them to 0 , this help reduce over-fitting (training only). After that another convolution layer is used. Also, another convolution layer is applied. After that there is pooling layer preceded by dropout layer and then there is a ninth layer for flattening the dataset that can be used for fully connected layer and then a dropout layer and then a connected layer followed by another dropout layer and finally we have output layer. In this algorithm the dropout percentage rate used is $25 \%$. The detailed specifications of these elements are given in Table 2. The suggested 15-layer CNN was built to classify segments of Electrocardiogram recordings of heart arrhythmia. The proposed network gives an auto categorization of input with no requireme nt for manual segmentation or annotation.
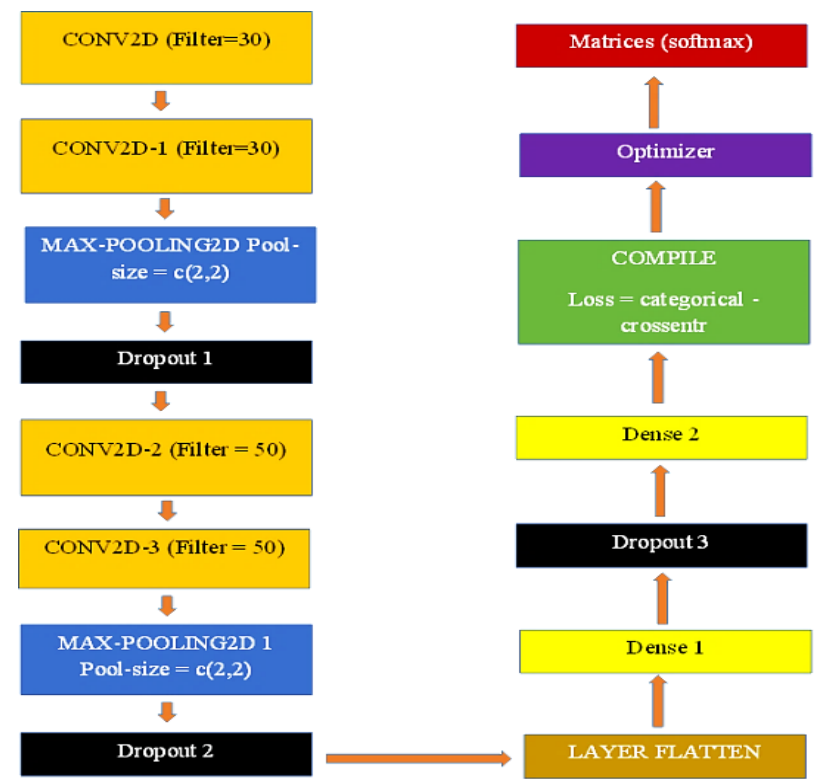

Figure 4. The layers of the proposed model for CNN 
Table 2. Summary model's layers for CNN

\begin{tabular}{cccc}
\hline Layer & Layer Name & shape & Layer Parameters \\
\hline 1 & conv2d & $(248,48,30)$ & 840 \\
2 & conv2d_1 & $(246,46,30)$ & 8130 \\
3 & max_pooling2d & $(123,23,30)$ & 0 \\
4 & dropout & $(123,23,30)$ & 0 \\
5 & conv2d_2 & $(121,21,50)$ & 13550 \\
6 & conv2d_3 & $(119,19,50)$ & 22550 \\
7 & max_pooling2d_1 & $(59,9,50)$ & 0 \\
8 & dropout_1 & $(59,9,50)$ & 0 \\
9 & flatten & (None, 26550) & 0 \\
10 & dense & (None, 100) & 2655100 \\
11 & dropout_2 & (None, 100) & 0 \\
12 & dense_1 & (None, 17) & 1717 \\
\hline
\end{tabular}

\section{RESULTS AND DISCUSSION}

Throughout this research, computer vision/deep learning model is created by using the $\mathrm{R}$ programming platform utilizing Keras and Tensorflow libraries to develop the proposed model. The dataset consists of 1000 fragments of ECG signals. It is divided into three groups, the training set accounting for $70 \%$ of the dataset, the validation set with $15 \%$, the testing set with $15 \%$. The input samples were converted into images. After training the 2D CNN, the model obtained overall accuracy of $96.67 \%$ for all class. Verification and training sets were chosen on a random basis. Figure 5 shows average the convergence of the proposed model in learning all 17 categories of cardiac arrhythmia during the training phase. The figure shows that the algorithm settles very quickly. It is shown here that at around 30 epochs the algorithm reaches the vicinity of its maximum accuracy of $96 \%$. The complexity of the algorithm in terms of time and space is reasonable with $(14 \mathrm{~m}$ and $29 \mathrm{sec})$ training time at $6 \mathrm{~GB}$ maximum memory usage. As for classification, with ( $4 \mathrm{~m}$ and $38 \mathrm{sec}$.) time at 2 GB maximum memory usage the algorithm is considered light-weight given the fact that it deals with $2 \mathrm{D}$ image data.

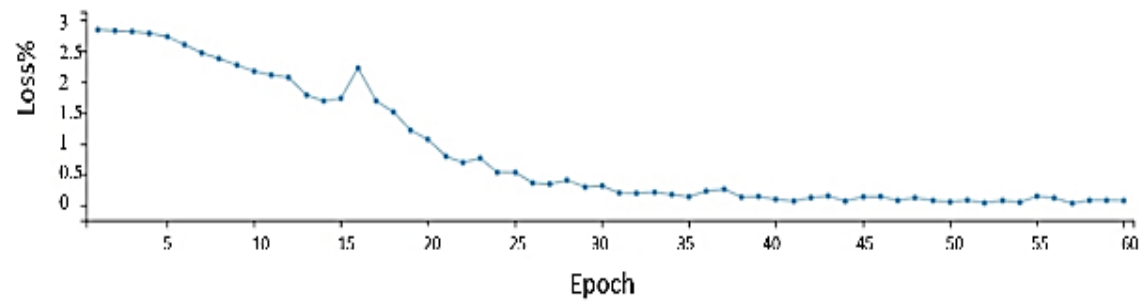

(a)

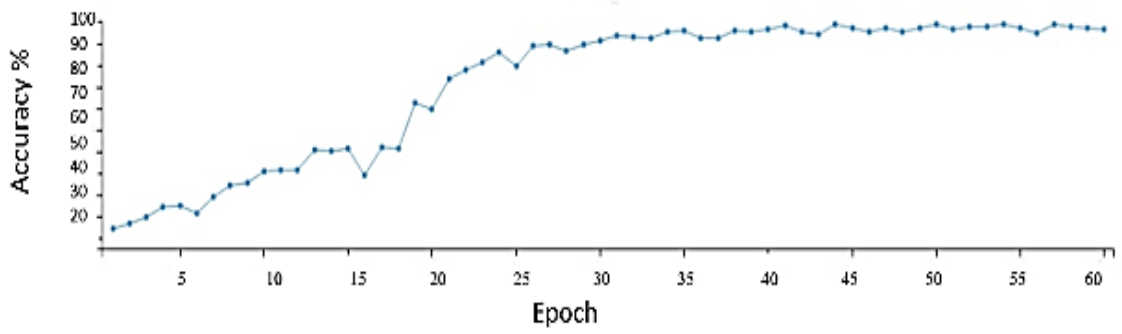

(b)

Figure 5. The convergence of the proposed model, (a) Los s function for training data,

(b) Accuracy for training data

Table 3 gives detailed comparison of the performance of the proposed method with other related research. In this table, it is seen that only three researchers consider all 17 classes with full length signals. Among those three, the proposed method produces the highest accuracy by high margin. The proposed algorithm also proves superior compared to the other approaches which consider less categories and shorter signals, albeit with lower margin. The table also shows clear lead for the deep learning methods and SVM over other machine learning approaches. It is obvious that the layout adopted here, as well as the decision to utilize 2-D images rather than 1-D signals gave clear advantage to this implementation as compared to other settings and choices made previously. Table 4 summarizes the performance metrics of the algorithm. 
Table 3. Comparison with other results in the literature

\begin{tabular}{|c|c|c|c|c|c|}
\hline Work & Year & Length of signal & No. of classes & Type of classifier & Accuracy \\
\hline Lin and Yang [18] & 2014 & 120 samples $(0.33 \mathrm{~s})$ & 5 & Weighted LD & $93 \%$ \\
\hline Huang et al. [19] & 2014 & 200 samples $(0.56 \mathrm{~s})$ & 5 & $\begin{array}{c}\text { Ensemble of SVM (Support } \\
\text { Vector Machines) }\end{array}$ & $94 \%$ \\
\hline Ye, Kumar, and Coimbra [21] & 2012 & 300 samples $(0.83 \mathrm{~s})$ & 5 & SVM & $86 \%$ \\
\hline Bazi, et al [22] & 2013 & 300 samples $(0.83 \mathrm{~s})$ & 5 & SVM, IWKLR, DT SVM & $92 \%$ \\
\hline Zhang and Luo [23] & 2014 & 227 samples $(0.63 \mathrm{~s})$ & 5 & Combined SVM & $87 \%$ \\
\hline Y1ldırım, et al [25] & 2018 & 3600 samples $(10 \mathrm{~s})$ & $\begin{array}{l}15 \\
17\end{array}$ & $1 \mathrm{D}-\mathrm{CNN}$ & $\begin{array}{l}92.51 \% \\
91.33 \%\end{array}$ \\
\hline Proposed method & 2019 & 3600 samples $(10 \mathrm{~s})$ & 17 & 2D-CNN & $96.673 \%$ \\
\hline
\end{tabular}

Table 4. Performance metrics of the proposed algorithm

\begin{tabular}{cccccc}
\hline sensitivity & specificity & precision & recall & F score & Overall AC \\
\hline $84.52 \%$ & $99.28 \%$ & $90.02 \%$ & $84.16 \%$ & $86.62 \%$ & $96.67 \%$ \\
\hline
\end{tabular}

\section{CONCLUSIONS}

This study's objective was to develop a 2D CNN deep learning model capable of successfully detecting a cardiovascular issue among 17 annotated categories of heart arrhythmia in long-term (10-s) Electrocardiograms. The proposed approach is shown to be: effective, quick (real-time ranking), comprehensive and can be integrated within other possibly wearable applications/devices. This $2 \mathrm{D}-\mathrm{CNN}$ system obtained a general ranking precision of $96,673 \%$ averaged for the 17 arrhythmia categories. An important finding here is that this algorithm is the best to date compared to its contemporaries. The results show that despite the use of big numbers of variables (up to 17 portions of cardiovascular illnesses) the algorithm managed to find discriminatory features and avoided local optima and overfitting. The results of the proposed algorithm states a strong case for adopting deep learning based automatic diagnosis techniques on larger scales in the healthcare systems. It also shows that these approach es can save lives by providing continuous and momentarily update about patients' states, this is of particular importance for vulnerable groups and in countries with weak presence of hospitals and healthcare institutions. It is also important in places where cardiologists are overloaded as it creates a framework for tracking and monitoring patients remotely without the need to book specialist clinic visits. The next step for this research can be the fusion of other data from sources other than ECG recordings to build more robust and comprehensive health diagnostic system. Such system can add the feature of case management which helps decide and schedule the subsequent stages of the health plans for patients.

\section{REFERENCES}

[1] D. Mozaffarian, et al., "Heart disease, stroke and research statistics at-a-glance," 2019.

[2] K. I. Ahmed, M. H. Habaebi, and M. R. Islam, "Smartphone aided real-time blood vein detection sy stem," Bulletin of Electrical Engineering and Informatics, vol. 8, no. 3, pp. 1096-1107, 2019.

[3] K. R. Siegersma, et al., "Artificial intelligence in cardiovascular imaging: state of the art and implications for the imaging cardiologist," Netherlands Heart Journal, vol. 27, no. 9, pp. 403-413, 2019.

[4] S. Mahmoodi, M. S. AL-Huseiny, and M. S. Nixon, "Similarity Registration for Shapes Based on Signed Distance Functions," Int. Symposium on Visual Computing, pp. 599-609, 2012.

[5] Z. Saringat, et al., "Comparative analy sis of classification algorithms for chronic kidney disease diagnosis," Bulletin of Electrical Engineering and Informatics, vol. 8, no. 4, pp. 1496-1501, 2019.

[6] T. J. Jun, et al., "ECG arrhythmia classification using a 2-D convolutional neural network," arXiv: Cornell Univeristy, 2018.

[7] S. Mahmoodi, "Discontinuity preserving method for noise removal of multi-carrier signals," Signal Processing, vol. 131, pp. 8-19, 2017.

[8] J. Mateo, et al., "An efficient method for ECG beat classification and correction of ectopic beats," Computers \& Electrical Engineering, vol. 53,pp. 219-229, 2016.

[9] R. G. Afkhami, G. Azarnia, and M. A. Tinati, "Cardiac arrhythmia classification using statistical and mixture modeling features of ECG signals," Pattern Recognition Letters, vol. 70, pp. 45-51, 2016.

[10] V. C. Chijindu, et al., "Detection of Prostate Cancer Using Radial/Axial Scanning of 2D Trans-rectal Ultrasound Images," Bulletin of Electrical Engineering and Informatics, vol. 7, no. 2, pp. 222-229, 2018.

[11] M. S. Al-Huseiny, S. Mahmoodi, and M. Nixon, "Level set gait analysis for synthesis and reconstruction," International Symposium on Visual Computing. Springer, pp. 377-386, 2009. 
[12] M. S. Al-Huseiny, S. Mahmoodi, and M. S. Nixon, "Robust rigid shape registration method using a level set formulation," Poceedings of the Sixth International Symposium on Visual Computing, pp. 252-261, 2010.

[13] G. B. Moody and R. G. Mark, "The impact of the MIT-BIH arrhythmia database," IEEE Eng Med Biol Mag, vol. 20 , no. 3, pp. 45-50, 2001.

[14] A. M. Khairuddin, K. N. F. Ku Azir, and P. Eh Kan, "A general framework for improving electrocardiograp hy monitoring system with machine learning," Bulletin of Electrical Engineering and Informatics, vol. 8, no. 1, pp. 261-268, 2019.

[15] J. Schmidhuber, "Deep learning in neural networks: An overview," Neural Networks, vol. 61, pp. 85-117, 2015.

[16] M. S. Al-Huseiny and S. Mahmoodi, "Shape registration using characteristic functions," IET Image Processing, vol. 9, no. 3, pp. 249-260, 2014.

[17] N. E. Md Isa, et al., "Motor imagery classification in Brain computer interface (BCI) based on EEG signal by using machine learning technique," Bulletin of Electrical Engineering and Informatics, vol. 8, no. 1, pp. 269-275, 2019.

[18] C. C. Lin and C. M. Yang, "Heartbeat Classification Using Normalized RR Intervals and Wavelet Features," 2014 International Symposium on Computer, Consumer and Control, Taichung, 2014, pp.650-653.

[19] H. Huang, et al., "A new hierarchical method for inter-patient heartbeat classification using random projections and RR intervals," Biomedical engineering online, vol. 13, no. 1, pp. 90-95, 2014.

[20] U. R. Acharya, et al., "A deep convolutional neural network model to classify heartbeats," Comput. Biol. Med., vol. 89, pp. 389-396, 2017.

[21] C. Ye, B. V. K. V. Kumar, and M. T. Coimbra, "Combining general multi-class and specific two-class classifiers for improved customized ECG heartbeat classification," Proceedings of the 21st International Conference on Pattern Recognition, pp. 2428-2431, 2012.

[22] Y. Bazi, N. Alajlan, H. AlHichri and S. Malek, "Domain adaptation methods for ECG classification," 2013 International Conference on Computer Medical Applications (ICCMA), Sousse, 2013, pp. 1-4.

[23] Z. Zhang, and L. Luo, "Heartbeat classification using decision level fusion," Biomedical Engineering Letters, vol. 4, no. 4, pp. 388-395, 2014.

[24] P. Pławiak, "Novel genetic ensembles of classifiers applied to myocardium dysfunction recognition based on ECG signals," Swarm and Evolutionary Computation, vol. 39, pp. 192-208, 2018.

[25] Ö. Yıldırım, et al., "Arrhythmia detection using deep convolutional neural network with long duration ECG signals," Computers in Biology and Medicine, vol. 102, pp.411-420, 2018.

\section{BIOGRAPHIES OF AUTHORS}
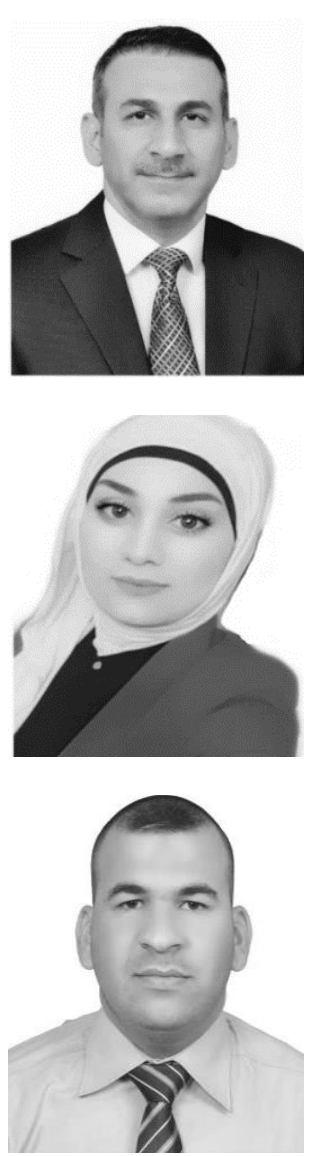

Muayed S AL-Huseiny received his BSc and MSc degrees in Computer and Software Engineering form AL-Mustansiriy a University in 2002 and 2005 respectively. He received PhD in Electronics and Computer Science from the University of Southampton in 2012.He received several research grants as well as study scholarship. He is an alumnus of the Fulbright Visiting Scholars program in the University of Central Oklahoma in 2014. Dr. AL-Huseiny is currently a faculty member and Dean of College of Computer Science and Information Technology in Wasit University. His research interests include computer vision, machine learning, biomedical engineering, IoT, and cryptography. He published several research papers in these fields and authored a book in cryptography. His current focus is on developing wearables for medical diagnosis by using machine learning.

Noor Khudhair Abbas received BSc degree in Electrical Engineering from Wasit University in 2016. She is currently doing MSc in Electrical Engineering. Her research interests include deep learning, $\mathrm{CNN}$, biomedical engineering, and computer vision.

Ahmed S Sajit is a faculty member at Wasit university; he received his BSc in Control and Computer Engineering from the University of Technology, Iraq, in 2010. He finished his MSc in Computer Engineering at California State University in Fullerton, USA in 2017. He was granted a nationally prestigious scholarship from the Prime minister's office in 2010. His research interests include: Computer Architecture, AI, Algorithms, and Phase Change Material Memory. 